\title{
Primes Differing by a Fixed Integer
}

\author{
By W. G. Leavitt and Albert A. Mullin
}

\begin{abstract}
It is shown that the equation $(*)(n-1)^{2}-\sigma(n) \phi(n)=m^{2}$ is always solvable by $n=p_{1} p_{2}$ where $p_{1}, p_{2}$ are primes differing by the integer $m$. This is called the "Standard" solution of $(*)$ and an $m$ for which this is the only solution is called a "*-number". While there are an infinite number of non *-numbers there are many (almost certainly infinitely many) *numbers, including $m=2$ (the twin prime case). A procedure for calculating all non *-numbers less than a given bound $L$ is devised and a table is given for $L=1000$.
\end{abstract}

The prime numbers $p_{1}, p_{2}$ are said to form a pair of "twin primes" if $p_{1}-p_{2}=2$. Using $\sigma(n)$, the sum of the divisors of $n$ (including $n$ itself), and $\phi(n)$, the number of numbers less than $n$ and relatively prime to $n, S$. A. Sergusov [1] has recently announced two criteria for an integer to be the product of twin primes. They are: $n$ is the product of twin primes if and only if either $\sigma(n)=n+1+2 \sqrt{n+1}$ or $\phi(n)=n+1-2 \sqrt{n+1}$. Combining these two results gives the sufficiency for:

THEOREM 1. The integer $n$ is the product of twin primes if and only if

$$
(n-1)^{2}-\sigma(n) \phi(n)=4 .
$$

Proof of the Necessity. For primes $p_{1}<p_{2}<\cdots<p_{k}$, suppose (1) is satisfied when $n=\Pi_{1}^{k} p_{i}^{n_{i}}$. Then (1) can be written

$$
2 \prod_{1}^{k} p_{i}^{n_{i}}+3=\prod_{1}^{k} p_{i}^{2 n_{i}}-\prod_{1}^{k}\left(p_{i}^{2 n_{i}}-p_{i}^{n_{i}-1}\right) .
$$

Since (2) would reduce for $k=1$ to $2 p^{n}+3=p^{n-1}$, it is clear that $k \geqslant 2$. Then note that if $p_{1}=2$, the left side of (2) is odd whereas the right side is even, and so $p_{1}>3$. Also from (2) it follows that if $p_{1}=3$, then $n_{1}=2$ or 1 , and in all other cases $n_{i}=1$.

Now if $k \geqslant 3$, it is easy to show that the right-hand side of (2) is greater than $p_{3} \Pi_{1}^{k} p_{i}^{n_{i}}$ and so exceeds the left-hand side, and if $k=2$ with $p_{1}=3$ and $n_{1}=2$, the right side is $3 p_{2}^{2}+78$ which again is always greater than the left-hand side.

In the only remaining case $k=2$ and $n_{1}=n_{2}=1$, so (2) reduces to $2 p_{1} p_{2}+3$ $=p_{1}^{2}+p_{2}^{2}-1$, that is $\left(p_{1}-p_{2}\right)^{2}=4$, and we conclude that $n=p_{1} p_{2}$ with $p_{1}-p_{2}$ $=2$.

We now generalize (1) to

$$
(n-1)^{2}-\sigma(n) \phi(n)=m^{2}
$$

for any integer $m$. It is easy to check that

THEOREM 2. If $n=p_{1} p_{2}$ with $p_{1}, p_{2}$ primes such that $p_{1}-p_{2}=m$, then $n$ satisfies (*).

Received December 4, 1980.

1980 Mathematics Subject Classification. Primary 10B99; Secondary 10A99. 
We will call the $n$ of Theorem 2 the standard solution of (*), and we will say that $m$ is $a^{*}$-number if $(*)$ has only the standard solution, that is if $(*)$ characterizes those $n$ which are products of two primes differing by the fixed integer $m$. Thus Theorem 1 states that 2 is a ${ }^{*}$-number.

THEOREM 3. For a given prime $p$, if $2 p-1$ is also prime, then $n=p^{k}(2 p-1)$ satisfies (*) for $m=p^{k}-1$, so $m=p^{k}-1$ is not $a^{*}$-number for all $k>2$. Similarly (*) has a solution $n=p^{k}(2 p+1)$ for $m=p^{k}+1$ whenever $p$ and $2 p+1$ are prime.

Proof. If $2 p \pm 1$ is prime, then for $n=p^{k}(2 p \pm 1)$ the left-hand side of $(*)$ becomes

$$
\left(p^{k}(2 p \pm 1)-1\right)^{2}-\left(p^{2 k}-p^{k-1}\right)\left(4 p^{2} \pm 4 p\right)=\left(p^{k} \pm 1\right)^{2}
$$

COROLlARY. There are an infinite number of odd non *-numbers and an infinite number of even non *-numbers.

Proof. This is clear since we have as non *numbers $2^{k}-1$ and $2^{k}+1$, and also $3^{k}-1$ and $3^{k}+1$ for all $k \geqslant 2$. Note: There are many other sequences of non *-numbers such as $7^{k}-1$ or $11^{k}+1$. Also note that except for 2 and 3 it is impossible for both $2 p-1$ and $2 p+1$ to be prime.

For primes $p_{1}<p_{2}<\cdots<p_{k}$ let

$$
f=\left(\prod_{1}^{k} p_{i}^{n_{i}}-1\right)^{2}-\prod_{1}^{k}\left(p_{i}^{2 n_{i}}-p_{i}^{n_{i}-1}\right),
$$

so that $n=\Pi_{1}^{k} p_{i}^{n_{i}}$ is a solution of $(*)$ if and only if $\sqrt{f}=m$ is an integer.

The next two propositions gave some limitations on the type of solutions that (*) may have.

Proposition 1. If $p$ is a prime such that $p \nmid m$ then the Mersenne number $M_{p}=2^{p}-1$ is not a solution of (*).

Proof. Let $n=M_{p}$ be a solution of (*). For a prime $q \mid M_{p}$, we have $2^{p} \equiv 1$ $(\bmod q)$ so $q \equiv 1(\bmod p)$. But then any $q^{2 r}-q^{r-1} \equiv 0(\bmod p)$ and also $M_{p}-1$ $\equiv 0(\bmod p)$. Thus from $(3)$ we have the contradiction $p^{2} \mid f$.

Proposition 2. If $p<q$ are primes, then $n=p q^{r}$ is not a solution of (*) for any $r \geqslant 2$ and any $m$.

Proof. If $n=p q^{r}$ is a solution of (*), then since $r>2$ we have $(q, m)=1$. Thus we can write $m=q^{t} h \pm \alpha$ for either $h=0$ or $(h, q)=1$ with some $t<1$, and some $0<\alpha \leqslant(q-1) / 2$. Thus $\alpha^{2} \equiv 1(\bmod q)$, so $\alpha^{2}=1$ and $(3)$ becomes

$$
q^{2 r}-2 p q^{r}+\left(p^{2}-1\right) q^{r-1}=q^{t} h\left(q^{t} h \pm 2\right) \text {. }
$$

Case 1. $p=2, q=3$. Then, since $p^{2}-1=3$, it follows from (4) that $t=r+1$. Thus (4) reduces to

$$
3^{r+1} h^{2} \pm 2 h-3^{r-1}+1=0
$$

But the left side of this equation is positive for all $h \geqslant 1$ and is nonzero for $h=0$. Thus no integral value of $h$ satisfies (4), so $m$ an integer is impossible. 
Case 2. In all other cases, since $q>p$, we have $q \nmid\left(p^{2}-1\right)$ and so $t=r-1$. Thus (4) becomes

$$
q^{r-1} h^{2} \pm 2 h-q^{r+1}+2 p q+1-p^{2}=0 .
$$

Writing the left side of this equation $F(h)$ we have, $F(0) \neq 0$, and clearly $F(h)$ is an increasing function for all $h \geqslant 1$. Since $q>p$, it is evident that $F(q)>0$. But also

$$
\begin{aligned}
F(q-1) & \leqslant q^{r-1}(q-1)^{2}+2(q-1)-q^{r+1}+2 p q+1-p^{2} \\
& \leqslant q^{r-1}(3-2 q)+p(2 q-p)-1 \\
& <q^{r-1}(3-2 q+2 q-p)-1=q^{r-1}(3-p)-1<0 .
\end{aligned}
$$

Thus $F(h)$ has no integral zeros, so again $m$ an integer is impossible.

Remark. The method of Theorem 1 can be used to show that, for certain values of $m,(*)$ has only the standard solution, so that $m$ is a *-number. However, with increasing $m$ the method rapidly becomes more complicated and must in any case be done one $m$ at a time. The following propositions yield a much simpler method, namely that for any chosen limit $L$ there is a systematic procedure by which all nonstandard solutions of (*) can be calculated for all $m \leqslant L$. Eliminating all such $m$ then leaves those ${ }^{*}$-numbers that are $\leqslant L$.

The following are clear from (3).

Proposition 3. If $k=1$, then $f<0$ so (*) is impossible.

Proposition 4. If $k \geqslant 2$, then $f$ is odd if and only if $n$ is even.

Proposition 5. In all cases $f$ is an increasing function of $n_{j}$ for all $j$.

Proof. We take the partial of $f$ with respect to $n_{j}$ and check directly in the case $j=1, k=2, n_{2}=1$ that the partial derivative is greater than $p_{1}^{n_{1}-1} \log p_{1}\left(p_{1}-p_{2}\right)^{2}$. In all other cases we examine the effect on the partial of replacing $p_{i}^{2 m_{1}}-p_{i}^{n_{1}-1}$ by $p_{i}^{2 n_{i}}$ for all $i \geqslant 2$ and (when $j>2$ ) replacing $2 p_{j}^{2 n_{j}}-p_{j}^{n_{j}-1}$ by $2 p_{j}^{2 n_{j}}$. It is then immediately clear that in all cases the partial derivative is positive.

Proposition 6. In the case $k=2$ and $n_{1}=1, f$ is a decreasing function of $p_{1}$ but is an increasing function of $p_{2}$. In all other cases $f$ is an increasing function of $p_{j}$ for all $j$.

Proof. When $k=2$ and $n_{1}=1$, we find that the partial derivative $f_{p_{1}}=$ $2 p_{2}^{n_{2}-1}\left(p_{1}-p_{2}\right)<0$. To show that all other partials are positive we examine (for the cases $k \geqslant 3$ or $k=2$ and $j \geqslant 2$ ) the effect of replacing in $f_{p_{j}}$ the term $2 n_{j} p_{j}^{2 n_{j}-1}-\left(n_{j}-1\right) p_{j}^{n_{j}-2}$ by $2 n_{j} p_{j}^{2 n_{j}-1}$ and replacing $p_{i}^{2 n_{j}}-p_{i}^{n-1}$ by $p_{i}^{2 n_{j}}$ for all $i \geqslant 2$ when $j \geqslant 2$, and for all $i \geqslant 3$ when $j=1$ and $k \geqslant 3$. Finally in the case $k=2$, $n_{1} \geqslant 2$ we show directly that

$$
f_{p_{1}} \geqslant p_{1}^{n_{1}-2} p_{2}^{n_{2}-1}\left[4 p_{1}^{3}+p_{2}^{2}-4 p_{1} p_{2}\right]>p_{1}^{n_{1}-2} p_{2}^{n_{2}-1}\left(2 p_{1}-p_{2}\right)^{2}
$$

Proposition 7. $f$ increases with $k$ in the sense that if $p$ is a prime not dividing a then $f\left(a p^{h}\right)>f(a)$ for all $h \geqslant 1$.

Proof. Let $b=\sigma(a) \phi(a)$. Then

$$
f\left(a p^{h}\right)=\left(a p^{h}-1\right)^{2}-b\left(p^{2 h}-p^{h-1}\right)>p^{2 h} f(a) .
$$


The Computations. In calculating nonstandard solutions $n=\Pi^{k} p_{i}^{n_{i}}$ of (*) it follows from Propositions 3 and 4 that $k \geqslant 2$ and if $k=2$ we do not need to consider the case $n_{1}=1$. Therefore from Propositions 5-7, we can regard $f$ as always an increasing function in all variables. Thus, for any upper limit $L$, there is clearly a systematic way of calculating for all $\sqrt{f}<L$, namely for each increasing $k$ (starting with $k=2$ ) and each increasing choice of the $n_{i}$ (starting with $n_{1}=2$ and $\left.n_{2}=1\right)$ we calculate for all $p_{1}<p_{2}<\cdots<p_{k}$ in each case up until $\sqrt{f}>$ $L$, recording all those $n$ in which $m=\sqrt{f}$ is an integer.

Note that in the following table we have separated the solutions for odd and even $m$ since the odd $m$ appear to have somewhat different properties. In fact, to say $m$ is an odd *-number is simply to say that $m+2$ is prime and (*) has the sole solution $n=2(m+2)$ or that (*) has no solutions at all.

The following is the set of all nonstandard solutions of $(*)$ for $m<1000$. Note that the solutions marked \# are those guaranteed by Theorem 3.

\begin{tabular}{|c|c|c|c|c|c|c|c|}
\hline $\mathrm{m}$ & $n$ & $\mathrm{~m}$ & $\mathrm{n}$ & m & $\mathrm{n}$ & $\mathrm{m}$ & $\mathrm{n}$ \\
\hline 3 & $2^{2} \cdot 3$ & 37 & $2^{2} \cdot 3^{3}$ & 163 & $2^{3} \cdot 3^{4}$ & 511 & $2^{9} \cdot 3 \#$ \\
\hline 5 & $2^{2} .5 \#$ & 49 & $2^{3} \cdot 5^{2}$ & 179 & $2 \cdot 3^{2} \cdot 19$ & 513 & $2^{9} .5 \#$ \\
\hline 7 & $2^{3} \cdot 3$ & 55 & $2^{3} \cdot 3^{3}$ & 185 & $2^{3} \cdot 3.19$ & 577 & $2.3^{2} \cdot 61$ \\
\hline 9 & $2^{3} \cdot 5$ & 61 & $2^{2} \cdot 3 \cdot 11$ & 249 & $2^{3} \cdot 5^{3}$ & 639 & $2.5 .11^{2}$ \\
\hline 13 & $2^{2} \cdot 11$ & 63 & $2^{6} \cdot 3 \#$ & 255 & $2^{8} \cdot 3$ & 739 & $2^{2} \cdot 3.131$ \\
\hline 15 & $2^{4} \cdot 3$ & 65 & $2^{6} .5 \#$ & 257 & $2^{8} .5$ \# & 813 & 2.7 .113 \\
\hline 17 & $2^{4} \cdot 5$ & 99 & 2.5 .19 & 303 & $2^{4} \cdot 109$ & 877 & 2.13 .67 \\
\hline 19 & $2^{3} \cdot 3^{2}$ & 127 & $2^{7} \cdot 3 \#$ & 321 & 2.5 .61 & 897 & $2^{7} \cdot 113$ \\
\hline 23 & $2^{3} .13$ & 129 & $2^{7} \cdot 5 \quad \#$ & 357 & $2^{2} .13 .19$ & 921 & $2^{3} \cdot 5.73$ \\
\hline 23 & 2.3 .7 & 145 & $2^{4} .53$ & 413 & $2^{2} \cdot 3^{2} \cdot 29$ & 955 & $2^{2} \cdot 3^{2} \cdot 67$ \\
\hline 31 & $2^{5} \cdot 3$ & 157 & $2^{2} \cdot 113$ & 437 & $2^{2} \cdot 311$ & 993 & $2^{5} \cdot 7.23$ \\
\hline 33 & $2^{5} \cdot 5$ & 159 & $2^{5} .41$ & 487 & $2^{3} \cdot 3^{5}$ & & \\
\hline
\end{tabular}

Note. The only values of $m \leqslant 5000$ for which (*) has a solution with $k=4$ are:

$\begin{array}{cc}m & n \\ 1744 & 3.5 .7 .41 \\ 3216 & 5.11 .13 .19 \\ 4516 & 3.5 .19 .41\end{array}$


EVEN

\begin{tabular}{|c|c|c|c|c|c|c|c|}
\hline $\mathrm{m}$ & $\mathrm{n}$ & $\mathrm{m}$ & $\mathrm{n}$ & $\mathrm{m}$ & $\mathrm{n}$ & $\mathrm{m}$ & $\mathrm{n}$ \\
\hline 8 & $3^{2} \cdot 5 \#$ & 172 & $3^{2} \cdot 7.11$ & 414 & $5^{2} \cdot 7.13$ & 694 & $3.5 .11^{2}$ \\
\hline 10 & $3^{2} \cdot 7 \#$ & 176 & 3.5 .31 & 432 & 7.17 .23 & 708 & 7.23 .29 \\
\hline 26 & $5^{2} .11 \#$ & 226 & $5^{3} .43$ & 438 & $19^{2} \cdot 79$ & 728 & $3^{6} .5 \#$ \\
\hline 26 & $3^{3} \cdot 5 \#$ & 228 & 7.11 .17 & 440 & $3^{2} \cdot 257$ & 730 & $3^{6} .7 \#$ \\
\hline 28 & $3^{3} \cdot 7 \#$ & 230 & $11^{2} \cdot 71$ & 440 & $7^{3} \cdot 47$ & 732 & $17^{2} \cdot 181$ \\
\hline 40 & 3.5 .7 & 240 & 5.13 .17 & 450 & 5.7 .53 & 744 & 13.19 .31 \\
\hline 46 & $5^{2} \cdot 23$ & 242 & $3^{4} \cdot 47$ & 456 & 5.19 .23 & 760 & 3.7 .101 \\
\hline 48 & $7^{2} \cdot 13 \#$ & 242 & $3^{5} \cdot 5$ & 472 & $11^{2} .149$ & 762 & 11.17 .37 \\
\hline 62 & $7^{2} \cdot 23$ & 244 & $3^{5} \cdot 7$ & 476 & $5^{3} .97$ & 796 & 3.5 .139 \\
\hline 78 & $7^{2} .31$ & 246 & 5.7 .29 & 510 & $7^{2} .199$ & 804 & 5.11 .67 \\
\hline 80 & $3^{4} .5 \#$ & 258 & $7^{2} .103$ & 516 & 5.11 .43 & 824 & $11^{2} \cdot 257$ \\
\hline 82 & $3^{3} .29$ & 288 & 7.13 .19 & 530 & $23^{2} \cdot 47 \#$ & 842 & $29^{2} .59 \#$ \\
\hline 82 & $3^{4} \cdot 7 \#$ & 296 & $5^{2} .137$ & 530 & $3^{2} \cdot 5 \cdot 43$ & 844 & 5.19 .43 \\
\hline 96 & 5.7 .11 & 320 & $11^{2} \cdot 101$ & 540 & $7^{3} .67$ & 870 & $11^{2} \cdot 271$ \\
\hline 118 & $3^{2} \cdot 71$ & 328 & 3.17 .19 & 620 & $3^{3} \cdot 11.13$ & 904 & 3.29 .31 \\
\hline 122 & $11^{2} \cdot 23 \#$ & 342 & $7^{2} \cdot 11^{2}$ & 626 & $5^{4} \cdot 11 \#$ & 926 & $5^{2} .419$ \\
\hline 126 & $5^{3} \cdot 11 \#$ & 342 & $7^{3} .13 \#$ & 648 & 13.17 .29 & 926 & $3^{2} \cdot 5^{2} \cdot 19$ \\
\hline 142 & 3.7 .19 & 354 & $5^{2} .163$ & 660 & 11.19 .29 & 932 & $7^{3} \cdot 131$ \\
\hline 144 & $11^{2} \cdot 37$ & 358 & $17^{2} \cdot 71$ & 662 & $13^{2} \cdot 191$ & 960 & $31^{2} .61 \#$ \\
\hline 148 & 3.11 .13 & 360 & $19^{2} \cdot 37 \#$ & 690 & $13^{2} .199$ & 990 & $23^{2} \cdot 199$ \\
\hline 166 & $11^{2} \cdot 47$ & 408 & 11.13 .23 & 692 & 7.13 .47 & 1000 & $3^{3} \cdot 7.29$ \\
\hline
\end{tabular}

Department of Mathematics and Statistics

University of Nebraska

Lincoln, Nebraska 68488

475-B Cook Drive

Redstone Arsenal, Alabama 35808

1. S. A. Sergusov, "On the problem of prime-twins," Jaraslav. Gos. Ped. Inst. Učen. Zap. Vyp. 82, Anal. i Algebra, 1971, pp. 85-86. (Russian) 